\title{
Family based therapy plus cognitive behavioural therapy (CBT) was better than CBT alone or family therapy alone for reducing adolescent drug abuse
}

Waldron HB, Slesnick N, Brody JL. Treatment outcomes for adolescent substance abuse at 4- and 7-month assessments.
J Consult Clin Psychol 2001 Oct;69:802-13.

QUESTION: Does cognitive behavioural therapy (CBT), functional family therapy (FFT), combined FFT and CBT, or a group psychoeducational intervention reduce adolescent substance abuse?

\section{Design}

Randomised (unclear allocation concealment*), unblinded*, controlled trial with 7 months of follow up.

\section{Setting}

A university centre in Albuquerque, New Mexico, USA.

\section{Patients}

120 adolescents who were $13-17$ years of age (mean age $16 \mathrm{y}, 80 \%$ boys), lived at home with a primary caretaker willing to participate in the study, and met DSM-IV criteria for a primary substance abuse disorder (primarily illicit drug use). Exclusion criteria were need for services beyond outpatient treatment, evidence of a psychotic or organic state, or a sibling in the study. Follow up was $88 \%$ at 4 months and $89 \%$ at 7 months.

\section{Intervention}

Adolescents were allocated to 1 of 4 groups: FFT ( $n=30)$, individual CBT (n=31), combined FFT and CBT $(n=29)$, or a group intervention $(n=30)$. FFT consisted of 12 weekly sessions of a systems oriented, behaviourally based family therapy with 2 phases: engaging families in treatment and enhancing motivation for change, and implementing behavioural changes in the family. Individual CBT consisted of motivational enhancement (2 sessions) and skills modules (10 sessions), including communication training, problem solving, peer refusal, and relapse prevention. The joint intervention consisted of weekly sessions of FFT and individual CBT. The group intervention provided education about substance abuse and its treatment and prevention (eight 90 min sessions).

\section{Main outcome measure}

Proportion of youths achieving minimal use (ie, use on $<10 \%$ of the days reported using the Timeline follow back interview).

\section{Main results}

A $4 \times 3$ repeated measures analysis of variance was done. At 4 months after treatment, FFT, joint FFT and CBT, and CBT alone showed reductions in adolescent marijuana use. At 7 months after treatment, FFT alone, joint FFT and CBT, and the group intervention showed reductions in marijuana use. When the FFT and joint conditions were combined for analysis, more adolescents in the combined family conditions than in the group condition changed from heavy to minimal marijuana use at 4 months $(\mathrm{p}<0.013)$ but the difference was not statistically significant at 7 months $(\mathrm{p}<0.128)$ (table). Combined family therapy plus CBT was better than CBT alone for increasing minimal use in adolescents at 7 months $(p<0.028)$ but the difference was not statistically significant at 4 months (table).

\section{Conclusion}

In adolescents with substance abuse, family based therapy, particularly in combination with cognitive behavioural therapy, showed better treatment outcomes for adolescents at 7 months after treatment.

*See glossary.
Source of funding: in part, National Institute

For correspondence: Dr H B Waldron, University of New Mexico, Albuquerque, NM, USA.

hwaldron@unm.edu on Drug Abuse.

Functional family therapy (FFT), cognitive behavioural therapy (CBT), combined FFT and CBT, or group intervention for adolescent substance abuse

\begin{tabular}{llllll} 
Outcome & Follow up & FFT & CBT & FFT + CBT & Group \\
$\begin{array}{c}\text { Minimal marijuana use (reported use } \\
\text { on }<10 \% \text { of } d \text { ) }\end{array}$ & 4 months & $45 \%$ & $28 \%$ & $44 \%$ & $12 \% \dagger$ \\
\hline & 7 months & $38 \%$ & $17 \% \dagger$ & $44 \%$ & $31 \%$ \\
\hline
\end{tabular}

†Comparison with combined family intervention was statistically significant.

\section{COMMENTARY}

In the past decade many studies have investigated the efficacy of various treatment models among adolescent substance abusers. Of the handful of promising models, only a few have been identified as exemplary. These models are appropriately theory driven, based on the previous identification of risk and protective factors for substance abuse, and are empirically supported by randomised controlled trials (RCTs). Family based models such as multidimensional family therapy and multisystemic therapy meet this standard of excellence. ${ }^{1}$ Other promising family, group, and individual based models include FFT, the more traditional 12 step model, motivational enhancement therapy, and CBT.

The study by Waldron et al represents the first systematic investigation of FFT in that it presents the results of an RCT with comparisons between FFT and other treatments. It provides evidence that FFT, particularly in combination with CBT, is efficacious in treating substance abusing adolescents. The long term persistence of the treatment effect remains to be determined. An interesting and unexpected finding was the positive effect at 7 months with the psychoeducational treatment group. This finding requires further study, given that it is less costly than the more intensive interventions.

Although the generalisability of the findings to the population at large is limited because the adolescents were primarily boys who had juvenile justice involvement, presented with marijuana abuse/dependency, and were treated in an outpatient setting, it is interesting to note that approximately $50 \%$ were Hispanic, which supports the effectiveness of this treatment model among this population.

In general, the results of this RCT show that FFT, particularly in combination with CBT, is efficacious for treating adolescents who meet $D S M-I V$ criteria for substance abuse. More specifically, this study shows the efficacy of this combined treatment approach among a population of adolescent male substance abusers (Hispanic and Anglo-American). However, further studies of FFT are warranted where larger samples are included, subgroups are identified within which this therapy is particularly efficacious, and site effects are examined.

Denise E Stevens, $\mathrm{PhD}$ MATRIX Public Health Consultants, Inc Killingworth, Connecticut, USA

1 National Institute on Drug Abuse. Principles of drug addiction treatment: a research-based guide. Rockville, MD: National Institute on Drug Abuse, National Institutes of Health, 2000 